\title{
TURAN'S INEQUALITY FOR APPELL POLYNOMIALS
}

SLAVKO SIMIC

Received 31 October 2003; Accepted 22 November 2003

We give some necessary and sufficient conditions for the class of Appell polynomials to satisfy well-known Turan's inequality. Among the other corollaries, we apply our results to some classes of orthogonal polynomials.

Copyright (c) 2006 Hindawi Publishing Corporation. All rights reserved.

\section{Introduction}

A real sequence $\left\{a_{n}\right\}, n=0,1,2, \ldots$ generates a sequence of Appell polynomials defined as:

$$
A_{n}(x):=\sum_{k=0}^{n} a_{k}\left(\begin{array}{l}
n \\
k
\end{array}\right) x^{n-k} ; \quad n=0,1,2, \ldots
$$

This class of polynomials is of importance in real and combinatorial analysis $[1,2]$. For example, the classical Bernoulli and Laguerre polynomials belong to Appell class.

It is said that the sequence of polynomials $\left\{C_{n}(x)\right\}$ have $T$ property if it satisfies Turan's inequality

$$
C_{n}^{2}(x)-C_{n-1}(x) C_{n+1}(x) \geq 0, \quad x \in[a, b], n \in N
$$

We will also consider inverse Turan's inequality and say that $\left\{C_{n}(x)\right\}$ have $T^{-}$property if it satisfies

$$
C_{n}^{2}(x)-C_{n-1}(x) C_{n+1}(x) \leq 0, \quad x \in[a, b], n \in N
$$

For $x \in[-1,1], T$ property was proved by Turán for Legendre's polynomials [5]; in [3] this property proved Szegö for Laguerre polynomials $(x \in R)$.

In 1964, Ikeda proved $T$ property for Bernoulli polynomials and $x \in[0,1]$.

Nowadays, the possibility of $T\left(T^{-}\right)$property for various classes of polynomials still attracts the attention of mathematicians. 
2 Turan's inequality for Appell polynomials

\section{Results}

Our task in this paper is to investigate $T$ property of Appell polynomials in two cases. In the first case we suppose that the generating sequence $\left\{a_{n}\right\}$ consists of positive numbers. In the second case we assume that all polynomials from the sequence $\left\{A_{n}(x)\right\}$ have real zeros only.

As concerns the first case, we can formulate the next theorem.

THeOREM 2.1. If the generating sequence $\left\{a_{n}\right\}$ consists of positive numbers only, then

(i) the sequence $\left\{A_{n}(x)\right\}$ have $T\left(T^{-}\right)$property for $x \in(0, b]$ if and only if the sequence $\left\{a_{n}\right\}$ have $T\left(T^{-}\right)$property;

(ii) the sequence $\left\{B_{n}(x)\right\}$ defined by

$$
B_{n}(x):=\left(A_{n}(x) / a_{n}\right)^{1 / n} ; \quad B_{0}(x):=1, \quad n \in N,
$$

is monotone nondecreasing (nonincreasing) for $x \in(0, b]$ if and only if the sequence $\left\{a_{n}\right\}$ have $T\left(T^{-}\right)$property.

From the above theorem follow some $T$ property criteria.

Proposition 2.2. If the sequence $\left\{a_{n}\right\}$ of positive numbers have not $T\left(T^{-}\right)$property then also $\left\{A_{n}(x)\right\}$ have not this property for $x \in[a, b], a \leq 0<b$.

Proposition 2.3. If the sequence $\left\{A_{n}(x) / a_{n}\right\}$ have $T^{-}(T)$ property for $x \in(0, b]$ then the sequence $\left\{A_{n}(x)\right\}$ have $T\left(T^{-}\right)$property for $x \in[0, \infty)$.

Proposition 2.4. Define

$$
A_{n}^{(\sigma)}(x):=\sum_{k=0}^{n} a_{k}^{\sigma}\left(\begin{array}{l}
n \\
k
\end{array}\right) x^{n-k}, \quad \sigma \in R ; \quad A_{n}^{(1)}(x)=A_{n}(x) .
$$

If $\left\{A_{n}(x)\right\}$ have $T$ property for $x>0$ then $\left\{A_{n}^{\sigma}(x)\right\}$ have $T$ property for $\sigma \geq 0$ and $T^{-}$property for $\sigma<0$.

Analogous statement takes place if $\left\{A_{n}(x)\right\}$ have $T^{-}$property.

$T$ property implies bounds for $A_{n}(x)$. Namely,

Proposition 2.5. If $\left\{A_{n}(x)\right\}\left(A_{0}(x)=a_{0}=1, a_{n}>0, n \in N\right)$ have $T$ property for $x \in$ $(0, b]$, then

$$
\frac{a_{n}}{a_{1}^{n}} \leq \frac{A_{n}(x)}{A_{1}^{n}(x)} \leq 1, \quad x \in[0, b], n \in N .
$$

If $\left\{A_{n}(x)\right\}$ have $T^{-}$property then the reverse inequalities hold.

In the sequel we will not assume positivity of the sequence $\left\{a_{n}\right\}$, but

Theorem 2.6. If all the zeros of polynomials $A_{n}(x), n \in N$ are real, then

(i) the generating sequence $\left\{a_{n}\right\}$ have T property;

(ii) the sequence $\left\{A_{n}(x)\right\}$ have $T$ property for $x \in R$. 
This theorem is particularly useful in the case of sequences of orthogonal polynomials. Because of orthogonality, all their zeros are real and, as a corollary of Theorem 2.6., we get

Proposition 2.7. The sequence of Hermite polynomials have T property for $x \in R$.

Denote by $\left\{L_{n}^{(a)}(x)\right\}$ the sequence of generalized Laguerre polynomials of order $a>-1$. Then

Proposition 2.8. The sequence $\left\{L_{n}^{(a)}(x) /\left(\begin{array}{c}n+a \\ n\end{array}\right)\right\}$ have T property for $x \in R$.

For $a=0$ we obtain Szego's theorem concerning Laguerre polynomials, mentioned above.

If the sequence of polynomials $\left\{Q_{n}(x)\right\}$ is not from Appell class, then the following assertion can be useful.

Theorem 2.9. If, for fixed $t \in[a, b]$, polynomials $R_{n}(x)$ defined by

$$
R_{n}(x):=\sum_{k=0}^{n} Q_{k}(t)\left(\begin{array}{l}
n \\
k
\end{array}\right) x^{n-k}, \quad n \in N,
$$

have all its zeros real, then the sequence $\left\{Q_{n}(x)\right\}$ have $T$ property for $x \in[a, b]$.

As a corollary we have the next

Proposition 2.10. If $\left\{P_{n}^{(\lambda)}(x)\right\}$ denotes the sequence of ultraspherical polynomials with parameter $\lambda>-1 / 2$, then the sequence $\left\{P_{n}^{(\lambda)}(x) / P_{n}^{(\lambda)}(1)\right\}$ have T property for $x \in[-1,1]$.

For $\lambda=1 / 2$ we obtain Turan's assertion on Legendre's polynomials and for $\lambda=1$ we proved $T$ property for the sequence $\left\{U_{n}(x) /(n+1)\right\}, x \in[-1,1]$, where $U_{n}(x)=P_{n}^{(1)}(x)$ is a Tchebychef polynomial of second kind.

\section{Proofs}

To prove part (i) of Theorem 2.1, suppose first $T\left(T^{-}\right)$property of the sequence $\left\{A_{n}(x)\right\}$ for $x \in(0, b]$. Then the polynomial $P(x)$,

$$
\begin{aligned}
P(x) & :=A_{n}^{2}(x)-A_{n-1}(x) A_{n+1}(x) \\
& =a_{n}^{2}-a_{n-1} a_{n+1}+(n-1)\left(a_{n} a_{n-1}-a_{n+1} a_{n-2}\right) x+\cdots+\left(a_{1}^{2}-a_{0} a_{2}\right) x^{2 n-2}
\end{aligned}
$$

is nonnegative (nonpositive) for $x \in(0, b]$.

Using the identity

$$
A_{n}^{\prime}(x)=n A_{n-1}(x)
$$

we obtain

$$
P^{\prime}(x)=(n-1)\left(A_{n}(x) A_{n-1}(x)-A_{n+1}(x) A_{n-2}(x)\right) .
$$

Hence, polynomials $P(x)$ and $P^{\prime}(x)$ are of the same sign, that is, $P(x)$ is either nonnegative and nondecreasing or nonpositive and nonincreasing for $x \in(0, b]$. 
4 Turan's inequality for Appell polynomials

Since it is also continuous in $x$, it follows that $P(0)=a_{n}^{2}-a_{n-1} a_{n+1}$ has the same sign as $P(x), x \in(0, b]$.

Suppose now that the sequence $\left\{a_{n}\right\}$ has $T\left(T^{-}\right)$property.

Putting $c_{n}=c_{n}(x):=a_{n} x^{-n}, x>0 ; n=0,1,2, \ldots$, we have to prove that $T\left(T^{-}\right)$property of $\left\{c_{n}\right\}$ implies the same property for the sequence $\left\{C_{n}\right\}$, where

$$
C_{n}:=\sum_{k=0}^{n}\left(\begin{array}{l}
n \\
k
\end{array}\right) c_{k} ; \quad n=0,1,2, \ldots
$$

It is easy to check that $T\left(T^{-}\right)$property of $\left\{c_{n}\right\}$ implies this property for the sequence $\left\{c_{n}^{(1)}\right\}$, defined by $c_{n}^{(1)}:=c_{n}+c_{n-1}$.

By induction, the same is valid for sequences $\left\{c_{n}^{(m)}\right\}$, where

$$
c_{n}^{(m+1)}:=c_{n}^{(m)}+c_{n-1}^{(m)}, \quad m=1,2, \ldots
$$

It is only left to note that $c_{n}^{(n)}=C_{n}$.

Remark 3.1. It follows that $T\left(T^{-}\right)$property of the sequence $\left\{a_{n}\right\}$ implies the same property of $\left\{A_{n}(x)\right\}$ for $x \in[0, \infty)$.

To prove part (ii) of Theorem 2.1, assume first that $\left\{a_{n}\right\}$ have $T$ property. By (i), the sequence $\left\{A_{n}(t)\right\}$ also have this property for $t>0$, that is,

$$
\frac{A_{n}(t)}{A_{n+1}(t)} \geq \frac{A_{n-1}(t)}{A_{n}(t)}
$$

or, by (3.2),

$$
\frac{1}{n+1} \frac{A_{n+1}^{\prime}(t)}{A_{n+1}(t)} \geq \frac{1}{n} \frac{A_{n}^{\prime}(t)}{A_{n}(t)} .
$$

Integrating (3.7) over $t \in[0, x]$, we get

$$
\left(A_{n+1}(x) / a_{n+1}\right)^{1 /(n+1)} \geq\left(A_{n}(x) / a_{n}\right)^{1 / n} ; \quad n=1,2, \ldots
$$

This means that the sequence $\left\{B_{n}(x)\right\}$ is monotone nondecreasing for each fixed $x>0$.

$T^{-}$case can be treated similarly.

Suppose now that $\left\{B_{n}(x)\right\}$ is monotone and consider the polynomial $Q(x)$ defined by

$$
Q(x):=\left(A_{n+1}(x) / a_{n+1}\right)^{n}-\left(A_{n}(x) / a_{n}\right)^{n+1} .
$$

By assumption, $Q(x)$ is nonnegative (nonpositive) for $x \in(0, b], b>0$.

We have $Q(0)=0$ and, by (3.2)

$$
Q^{\prime}(0)=n(n+1)\left(a_{n} / a_{n+1}-a_{n-1} / a_{n}\right) ; \quad n=1,2, \ldots
$$

Therefore,

$$
Q(x)=n(n+1)\left(a_{n} / a_{n+1}-a_{n-1} / a_{n}\right) x+\cdots+\left(\left(a_{0} / a_{n+1}\right)^{n}-\left(a_{0} / a_{n}\right)^{n+1}\right) x^{n(n+1)} \text {. }
$$


Since $x$ is independent of $n$, we see from (3.11) that, for sufficiently small positive $x$, the signs of $Q(x)$ and $Q^{\prime}(0)$ have to be the same, that is, the part (ii) is also proved.

Proof of Proposition 2.2. This is a consequence of the assertion (i) of Theorem 2.1.

Proof of the next proposition needs the following lemma.

Lemma 3.2. If the sequence $\left\{b_{n}\right\}, b_{0}:=1$ of positive numbers have $T\left(T^{-}\right)$property, then the sequence $\left\{b_{n}^{1 / n}\right\}$ is nonincreasing (nondecreasing).

Proof. T property implies $b_{n}^{2} \geq b_{n-1} b_{n+1}, n \in N$. Hence

$$
\left(b_{0} b_{2}\right)\left(b_{1} b_{3}\right)^{2}\left(b_{2} b_{4}\right)^{3} \cdots\left(b_{n-1} b_{n+1}\right)^{n} \leq b_{1}^{2} b_{2}^{4} b_{3}^{6} \cdots b_{n}^{2 n} .
$$

This gives $b_{n+1}^{n} \leq b_{n}^{n+1}$, that is, $\left\{b_{n}^{1 / n}\right\}$ is nonincreasing.

Proof of $T^{-}$case goes along the same lines.

Proof of Proposition 2.3. Assume that $\left\{A_{n}(x) / a_{n}\right\}$ have $T^{-}$property for $x \in(0, b]$. Then Lemma 3.2 asserts $\left\{\left(A_{n}(x) / a_{n}\right)^{1 / n}\right\}$ nondecreasing for $x \in(0, b]$. By part (ii) of Theorem 2.1 this implies $T$ property for $\left\{a_{n}\right\}$ which in turn, by part (i) and Remark 3.1, gives $T$ property of $\left\{A_{n}(x)\right\}$ for $x \in[0, \infty)$.

Proof of Proposition 2.4. This is a consequence of Theorem 2.1, part (i) and the fact that, if $\left\{a_{n}\right\}$ have $T$ property, then $\left\{a_{n}^{\sigma}\right\}$ have $T$ property for $\sigma \geq 0$ and $T^{-}$property for $\sigma<0$.

Proof of Proposition 2.5. T property and Lemma 3.2 imply $\left\{\left(A_{n}(x)\right)^{1 / n}\right\}$ nonincreasing. Therefore $A_{n}(x)^{1 / n} \leq A_{1}(x)$.

On the other hand, by Theorem 2.1 part (i), $T$ property of $\left\{A_{n}(x)\right\}$ implies $T$ property for $\left\{a_{n}\right\}$ which in turn, by part (ii), implies that the sequence $\left\{\left(A_{n}(x) / a_{n}\right)^{1 / n}\right\}$ is nondecreasing. Hence

$$
\left(A_{n}(x) / a_{n}\right)^{1 / n} \geq A_{1}(x) / a_{1} ; \quad x>0, n=1,2, \ldots
$$

This is exactly the left-hand side of the inequality from Proposition 2.4.

The other case can be treated similarly.

Proof of Theorem 2.6. We will prove first the part (ii) of this theorem. For this purpose, let

$$
A_{n+1}(x)=a_{0} \prod_{i=0}^{n}\left(x-x_{i}\right), \quad x_{i} \in R, n=0,1,2, \ldots
$$

Taking logarithmic derivative, by (3.2), we get

$$
(n+1) \frac{A_{n}(x)}{A_{n+1}(x)}=\frac{A_{n+1}^{\prime}(x)}{A_{n+1}(x)}=\sum_{i=0}^{n} \frac{1}{x-x_{i}} .
$$

Analogously,

$$
(n+1) \frac{n A_{n-1}(x) A_{n+1}(x)-(n+1) A_{n}^{2}(x)}{A_{n+1}^{2}(x)}=\left(\frac{A_{n+1}^{\prime}(x)}{A_{n+1}(x)}\right)^{\prime}=-\sum_{i=0}^{n} \frac{1}{\left(x-x_{i}\right)^{2}} .
$$


6 Turan's inequality for Appell polynomials

Therefore, by Cauchy's inequality, we obtain

$$
\begin{aligned}
\frac{A_{n}^{2}(x)}{A_{n+1}^{2}(x)} & =\left(\frac{1}{n+1} \sum_{i=0}^{n} \frac{1}{x-x_{i}}\right)^{2} \leq \frac{1}{n+1} \sum_{i=0}^{n} \frac{1}{\left(x-x_{i}\right)^{2}} \\
& =\frac{(n+1) A_{n}^{2}(x)-n A_{n-1}(x) A_{n+1}(x)}{A_{n+1}^{2}(x)}
\end{aligned}
$$

which is equivalent to $T$ property for the sequence $\left\{A_{n}(x)\right\}, x \in R$.

Since $a_{n}=A_{n}(0)$, the assertion (i) follows from (ii) for $x=0$.

Proof of Proposition 2.7. The classical Hermite polynomials $\left\{H_{n}(x)\right\}$ are defined by [4, page 105],

$$
\frac{H_{n}(x)}{n !}=\sum_{i=0}^{[n / 2]} \frac{(-1)^{i}}{i !} \frac{(2 x)^{n-2 i}}{(n-2 i) !}, \quad n=0,1,2, \ldots
$$

To see that the sequence $\left\{H_{n}(x / 2)\right\}$ belongs to the Appell class, write (3.18) in the form

$$
H_{n}(x / 2)=\sum_{i=0}^{n} h_{i}\left(\begin{array}{l}
n \\
i
\end{array}\right) x^{n-i}
$$

where

$$
h_{2 k}=\frac{(-1)^{k}(2 k) !}{k !} ; \quad h_{2 k+1}=0 ; \quad k=0,1,2, \ldots
$$

Since all their zeros are real [4, page 110], by Theorem 2.6, part (ii), $T$ property follows for $x \in R$.

Proof of Proposition 2.8. The class of generalized Laguerre polynomials $\left\{L_{n}^{(a)}(x)\right\}$ of order $a>-1$ is defined by [4, page 100]

$$
L_{n}^{(a)}(x)=\sum_{i=0}^{n}\left(\begin{array}{c}
n+a \\
n-i
\end{array}\right) \frac{(-x)^{i}}{i !}
$$

All its zeros are real and positive [4, page 110]. For $x \neq 0$, an elementary transform gives

$$
x^{n} L_{n}^{(a)}(1 / x) /\left(\begin{array}{c}
n+a \\
n
\end{array}\right)=\Gamma(a+1) \sum_{i=0}^{n} \frac{(-1)^{i}}{\Gamma(i+a+1)}\left(\begin{array}{c}
n \\
i
\end{array}\right) x^{n-i} .
$$

Now, we can apply Theorem 2.6, part (ii).

Proof of Theorem 2.9. This assertion is a consequence of Theorem 2.6, part (i).

Proof of Proposition 2.10. An explicit form of ultraspherical polynomials of order $\lambda$ is the following [4, page 85]

$$
P_{n}^{(\lambda)}(x)=\sum_{i=0}^{[n / 2]}(-1)^{i} \frac{\Gamma(n-i+\lambda)}{\Gamma(\lambda) \Gamma(i+1) \Gamma(n-2 i+1)}(2 x)^{n-2 i} .
$$


All its zeros are real and belong to the interval $(-1,1)$. We also have

$$
P_{n}^{(\lambda)}(1)=\left(\begin{array}{c}
n+2 \lambda-1 \\
n
\end{array}\right)=(-1)^{n} P_{n}^{(\lambda)}(-1)
$$

[4, page 81$]$.

For the proof we will use the following identity [4, page 384]:

$$
\sum_{k=0}^{n} \frac{P_{k}^{(\lambda)}(t)}{P_{k}^{(\lambda)}(1)}\left(\begin{array}{l}
n \\
k
\end{array}\right) x^{n-k}=\left(1+2 t x+x^{2}\right)^{n / 2} \frac{P_{n}^{(\lambda)}\left[\left(1+2 t x+x^{2}\right)^{-1 / 2}(t+x)\right]}{P_{n}^{(\lambda)}(1)} .
$$

Taking $t \in(-1,1)$ we can see that all the zeros $\left\{x_{i}\right\}$ of the polynomial on the right-hand side of (3.25) are real and given by

$$
x_{i}=t_{i} \sqrt{\frac{1-t^{2}}{1-t_{i}^{2}}}-t
$$

where $\left\{t_{i}\right\}$ are corresponding zeros of $P_{n}^{(\lambda)}(t)$.

Hence, by (3.24) and Theorem 2.9, we obtain the assertion of Proposition 2.10.

\section{References}

[1] P. A. MacMahon, Combinatory Analysis, Two Volumes (bound as one), Chelsea Publishing, New York, 1960.

[2] J. Riordan, An Introduction to Combinatorial Analysis, Wiley Publications in Mathematical Statistics, John Wiley \& Sons, New York; Chapman \& Hall, London, 1958.

[3] G. Szegö, On an inequality of P. Turán concerning Legendre polynomials, American Mathematical Society Bulletin 54 (1948), 401-405.

[4] _ Orthogonal Polynomials, Revised ed., American Mathematical Society Colloquium Publications, vol. 23, American Mathematical Society, Rhode Island, 1959.

[5] P. Turán, On the zeros of the polynomials of Legendre, Československá Akademie Věd. Časopis Pro Pěstování Matematiky 75 (1950), 113-122.

Slavko Simic: Mathematical Institute SANU, Kneza Mihaila 35, 11001 Belgrade, Serbia and Montenegro

E-mail address: ssimic@turing.mi.sanu.ac.yu 